\title{
BMJ Open Suicidal behaviour in adults during the COVID-19 pandemic: protocol for systematic review of observational studies
}

\author{
Fernando José Guedes da Silva Junior (D) , ${ }^{1}$ Priscilla Ingrid Gomes Miranda (D) ,1 \\ Jaqueline Carvalho e Silva Sales, ${ }^{1}$ Adriana da Cunha Menezes Parente, ${ }^{1}$ \\ Claudete Ferreira de Souza Monteiro, ${ }^{1}$ Ana Paula Cardoso Costa, ${ }^{1}$ \\ Luana Ruth Braga Campos, ${ }^{1}$ Larissa Alves de Araújo Lima, ${ }^{1}$ \\ Aline Raquel de Sousa Ibiapina, ${ }^{1}$ Sandra Cristina Pillon, ${ }^{2}$ \\ Angélica Martins de Souza Gonçalves, ${ }^{3}$ Chrystiany Plácido de Brito Vieira, ${ }^{1}$ \\ Francisca Tereza de Galiza ${ }^{1}$
}

To cite: Silva Junior FJGda, Miranda PIG, Sales JCeS, et al. Suicidal behaviour in adults during the COVID-19 pandemic: protocol for systematic review of observational studies. BMJ Open 2021;11:e045313. doi:10.1136/ bmjopen-2020-045313

- Prepublication history for this paper is available online. To view these files, please visit the journal online (http://dx.doi. org/10.1136/bmjopen-2020045313).

Received 28 September 2020 Accepted 27 July 2021

A) Check for updates

(c) Author(s) (or their employer(s)) 2021. Re-use permitted under CC BY-NC. No commercial re-use. See rights and permissions. Published by BMJ.

${ }^{1}$ Nursing Department, Universidade Federal do Piaui, Teresina, Piauí, Brazil

${ }^{2}$ Nursing Department, USP, Ribeirão Preto, São Paulo, Brazil

${ }^{3}$ Nursing Department, UFSCar, Sao Carlos, São Paulo, Brazil

Correspondence to Dr Fernando José Guedes da Silva Junior;

fernandoguedes@ufpi.edu.br

\section{ABSTRACT}

Introduction COVID-19 pandemic has an impact on mortality indicators worldwide. Mitigation and repression actions to reduce the morbidity and mortality associated with the disease are necessary. However, they are criticised in the economic, social and psychological spheres. This social isolation, increased unemployment, routine changes, news of health complications and deaths related to COVID-19 can cause psychological repercussions that will certainly intensify in the coming months, and suicidal behaviour presents itself as a fatal outcome. It is necessary to know factors associated with suicidal behaviour in adults during the pandemic. Although there are studies, there is no systematic review to assess these factors, specifically in adults. The objective is to critically synthesise the scientific evidence on the factors associated with suicidal behaviour in adults in the COVID-19 pandemic.

Methods and analyses A systematic review will be carried out, recommended by the Preferred Reporting Items for Systematic Reviews and Meta-Analyses (PRISMA) protocol, in seven databases: Medical Literature Analysis and Retrieval System Online (MEDLINE), ISI of Knowledge, Excerpta Medica Database (EMBASE), SCOPUS, Latin American and Caribbean Health Sciences Literature (LILACS), Chinese National Knowledge Infrastructure (PsycINF0), Chinese National Knowledge Infrastructure (CNKI) and ScienceDirect. Preliminary search was carried out on 30 July 2020 and will be updated in March 2021. No restrictions on publication date, study location or languages will be considered in this review. The preliminary research strategies were carried out on 30 July 2020 and will be updated in February 2021. To measure the agreement between reviewers at each screening stage, Cohen's Kappa will be calculated. Primary outcome will be factors related to suicidal behaviour in adults during COVID-19 pandemic. Grouped standardised mean differences and $95 \%$ Cls will be calculated. The risk of bias in observational studies will be assessed using the Methodological Index for Non-Randomised Studies (MINORS). Statistical heterogeneity will be assessed with the I2 statistic.

\section{Strengths and limitations of this study}

- This systematic review protocol aims to reduce duplication of studies, strengthen the transparency of the method and process adopted in conducting it, in addition to enabling peer review and reducing bias.

- This protocol is an initial step to provide scientific evidence to support decision-making in the context of health services and policy management to face the impact of this global problem and its impacts, including on suicidal behaviour in adults.

- As limitations, the following stand out: possible underreporting of suicidal behaviour records, as it is an outcome involving stigmas; the heterogeneity of the studies as well as the methodological assessment and probably the small number of studies, considering that the COVID-19 pandemic is recent, and its implications are still being investigated.

Ethics and disclosure Ethical approval is not required, as primary data will not be collected. The findings will be disseminated through peer-reviewed publications. PROSPERO registration number CRD42020208816.

\section{INTRODUCTION}

A new virus of the Coronaviridae family (SARS-CoV-2) was identified in Wuhan, China, at the end of 2019 and is responsible for the onset of the COVID-19. This infection can present from asymptomatic to severe clinical conditions. Its symptoms can vary from cold, influenza syndrome or viral pneumonia. Due to its high pathogenicity, people around the world were infected and died from the disease. ${ }^{1}$ According to the WHO, it is estimated that 97464094 people worldwide were infected and 2112689 died due to COVID19, until 24 January 2021, which shows the 
severity of the pandemic, considered a public calamity by several nations. ${ }^{2}$

In this perspective, countless countries have sought to take actions to mitigate the emergence of new cases, to reduce the morbidity and mortality associated with the disease, and avoid their side effects on health systems around the world. ${ }^{3}$

These actions are part of the pandemic response plan divided into containment, mitigation, suppression and recovery. In containment, cases are observed and recorded in an information system. In mitigation, vertical isolation is carried out through the closure of agglomeration sites. In suppression, there is horizontal isolation, in which any contact is avoided, and all people stay in their homes, leaving only in cases of emergency until the vaccine is obtained. Finally, recovery is the involution of the pandemic until it is residual. ${ }^{4}$

In this sense, the nations adopted the mitigation and suppression phases, with vertical and horizontal isolations. These measures, although necessary, present criticisms in the areas: economic, social and psychological. People have been afraid of getting sick, they feel insecure about the future, but they need to isolate themselves since social contact must be minimal. This attitude has led to the emergence of psychological distress and suicidal behaviour in adults worldwide. ${ }^{45}$

Suicidal behaviour includes suicidal ideation, attempted suicide and death by suicide. ${ }^{6}$ It is considered a public health problem, and with the COVID-19 pandemic, it is in evidence. Social isolation, increased unemployment, routine changes, news from close people hospitalised in intensive care beds and deaths have generated emotional stress and reflected increased ideas and attempts to end their own lives. ${ }^{7}$ This reality is shared even by people without serious mental disorders, which demonstrates that the risk of suicide is considered high due to the high levels of anguish commonly experienced in times of pandemic. ${ }^{8}$

It is necessary to pay attention to this problem with care aimed not only at contagion by SARS-Cov-2 but also with the consequences associated with the infection. Suicidal behaviour is often debilitating for those who fail to reach the end of the action, but it also affects families. Thus, measures must be taken at various healthcare levels to identify people at risk for this behaviour earlier. However, studies on its impact on suicidal behaviour in adults are still scarce. In this perspective, given the Preferred Reporting Items for Systematic Reviews and Meta-Analyses Protocols (PRISMA-P), we intend to carry out a systematic and reproducible search strategy to consult the impact of the COVID-19 pandemic on suicidal behaviour in adults in the literature.

\section{RESEARCH OBJECTIVES}

This systematic review aims to critically synthesise the scientific evidence on the factors associated with suicidal behaviour in adults in the COVID-19 pandemic.

\section{METHODS AND ANALYSES}

\section{Search strategy}

A systematic and reproducible search strategy will be carried out to optimise methodological transparency, using the PRISMA-P checklist. ${ }^{9}$ The acronym PICOS (Population/Intervention/Comparison/Outcomes/ Study Design $)^{10}$ was used to support the elaboration of the research question in this review, in order to ensure the systematic search in the scientific literature: 'What are the factors associated with suicidal behaviour in adults in the COVID-19 pandemic?'.

Studies will be retrieved from the seven electronic bibliographic databases: Medical Literature Analysis and Retrieval System Online (MEDLINE) via PubMed, ISI of Knowledge via Web of Science, Excerpta Medica Database (EMBASE), SCOPUS, Latin American and Caribbean Health Sciences Literature (LILACS), ScienceDirect and Chinese National Knowledge Infrastructure (CNKI) and Psychology Information (PsycINFO). No restrictions on publication date, study location or languages will be considered in this review. Additionally, a secondary search will be conducted on Google Scholar. The reference section of the included studies will be searched manually to identify additional studies.

For search, only the key terms will be used according to the PICOS acronym. The bibliographic software EndNote (https://www.myendnoteweb.com/) will be used to store, organise and manage all references and ensure a systematic and comprehensive search.

Initially, the strategy to search for the studies will consist of the combination of controlled descriptors (indexed in the respective databases) and uncontrolled descriptors, according to the indication of each base through the combination of controlled and uncontrolled descriptors, through Boolean operators 'AND' and 'OR'. ${ }^{11}$ (table 1).

The preliminary search strategies were carried out on 30 July 2020 and will be updated in March 2021. Besides, this systematic review is expected to be completed in June 2021.

\section{Study selection}

A summary of the PICOS acronym is shown in table 2.

Only observational studies investigating the factors associated with suicidal behaviour in adults ( $\geq 18$ years old $)$ in the COVID-19 pandemic will be included in this review. Studies carried out with children, adolescents, pregnant women, older people, health professionals and students will be excluded. Those who reported factors associated with suicidal behaviour in other pandemics, RCNs (nonrandomized clinical trials), randomised controlled trials, systematic reviews, qualitative studies and grey literature will also be excluded. In this systematic review, there are no restrictions on languages.

\section{Screening and data extraction}

The initial screening of studies will be carried out based on the information in their titles and abstracts by two independent researchers (FJGdSJ and JCeSS). When the 
Table 1 Concepts and search items

\begin{tabular}{|c|c|}
\hline Databases & Search items \\
\hline MEDLINE & \#1 ((((((“adult”(MeSH Terms)) OR “adult” (All fields) OR “adults”(All Fields) OR “adults age”(All Fields))) \\
\hline ISI of Knowledge & \#2 ((“Coronavirus"(MeSH Terms] OR "Coronavirus"(All Fields)) OR ("COVID-19”(All Fields] OR "Severe \\
\hline SCOPUS & Syndrome Coronavirus 2"(All Fields] OR "2019-nCoV"(All Fields] OR "SARS-CoV-2"(All Fields)) OR \\
\hline LILACS & "Pandemics"(MeSH Terms)) \\
\hline PsycINFO & \#3 ((((“suicide”(MeSH Terms)) OR “suicide” (All Fields)) OR “suicides” (All Fields)) OR "suicidal \\
\hline
\end{tabular}

CNKI, Chinese National Knowledge Infrastructure; EMBASE, Excerpta Medica Database; LILACS, Latin American and Caribbean Health Sciences Literature; MEDLINE, Medical Literature Analysis and Retrieval System Online; PsycINFO, Psychology Information.

reviewers disagree, the study will be assessed and, if the disagreement persists, a third reviewer (CFdSM) will make the final decision. Then, the screening will be performed with the reading of the full text, which is also performed by the same independent researchers. To measure the agreement between reviewers at each screening stage, Cohen's Kappa will be calculated.

Once consensus has been reached on the selected studies, a standardised form based on previous studies ${ }^{1213}$ will be used for data extraction. The information extracted will be bibliometric characteristics (title of the study, the title of the journal, impact factor, authors, country, language, publication year, host institution of the study, conflict of interest and promotion); methodological characteristics (study design, research objective or question or hypothesis, sample characteristics, recruitment methods and study completion rates, declared duration of follow-up, statistical analysis); main findings and implications for clinical practice; and conclusions. ${ }^{12} 13$ The same two reviewers will perform data extraction independently. Discrepancies between reviewers will be resolved by discussion or, in the absence of agreement, by a third reviewer (CFdSM).

\section{Missing data management}

The researchers will contact the original author to obtain the missing or incomplete data. We will wait 15 days after sending an email to receive a response. If we are unable to obtain the missing data, incomplete data will be excluded from the analysis.

\section{Methodological appraisal}

The internal validity and risk of bias for non-randomised studies, the Methodological Index for Non-Randomised Studies (MINORS) ${ }^{14}$ will be used. This MINORS instrument contains eight items for observational studies: (1) a clearly stated aim, (2) inclusion of consecutive patients, (3) prospective collection of data, (4) endpoints appropriate to the study's objective, (5) unbiased assessment of the study endpoint, (6) follow-up period appropriate to the study's objective, (7) loss to follow-up less than $5 \%$ and (8) prospective calculation of the study size. ${ }^{14}$ All items from the MINORS tool will be rated from 0 to 2, which means the score 0 indicates that the information was not reported, 1 indicates that the information was inadequately reported and 2 that the information was adequately reported. ${ }^{14}$ The same two reviewers (FJGdSJ

\begin{tabular}{|c|c|c|}
\hline PICOS acronym $^{10}$ & Inclusion criteria & Exclusion criteria \\
\hline P-Population & $\begin{array}{l}\text { Adults of both genders. } \\
\text { Age } \geq 18 \text { years old and of any ethnicity }\end{array}$ & $\begin{array}{l}\text { Children, adolescents, pregnant women, } \\
\text { older people, health professionals and } \\
\text { students of both genders }\end{array}$ \\
\hline I-Intervention/Exposure & $\begin{array}{l}\text { The impact of the COVID-19 pandemic on suicidal } \\
\text { behaviour }\end{array}$ & Other previous pandemics \\
\hline C-Comparison & Not applicable & - \\
\hline O-Outcome & $\begin{array}{l}\text { The primary outcomes are the prevalence of suicidal } \\
\text { ideation, attempt, suicide mortality and related } \\
\text { factors during the pandemic }\end{array}$ & $\begin{array}{l}\text { Studies reporting the prevalence of suicidal } \\
\text { behaviour among adults due to other } \\
\text { pandemics }\end{array}$ \\
\hline S-Study design & Observational studies & $\begin{array}{l}\text { RCT, NRCT, systematic reviews, qualitative } \\
\text { studies and grey literature }\end{array}$ \\
\hline Language & All languages & None \\
\hline Setting & All settings & None \\
\hline
\end{tabular}

NRCT, non-randomised controlled trials; RCT, randomised controlled trials. 
and JCeSS) will perform the critical appraisal independently. Disagreements will be resolved by a third reviewer (CFdSM). The inter-rater reliability will be rated using intraclass correlation coefficients. ${ }^{15}$ The authors from the original articles will be contacted if additional information is required.

\section{Assessment of publication bias}

To assess the publication bias, a funnel plot will be examined. Following the approach proposed by Duval and Tweedie,${ }^{16}$ the number of studies that are missing from the funnel plot will be estimated, if any. The effect size after the imputation of these missing studies will be estimated by the trim-and-fill method. ${ }^{16}$ Egger's test will also be performed. ${ }^{17}$

\section{Heterogeneity assessment}

Statistical heterogeneity will be assessed with the I2 statistic. $^{18}$ An I2 statistic of less than $50 \%$ indicates a low level of statistical heterogeneity; $50 \%$ or more will be considered substantial statistical heterogeneity. If I2 $\geq 50 \%$ is identified, we will explore a possible subgroup analysis.

\section{Data synthesis}

After performing the data synthesis and categorising the studies, the final report will be prepared according to the PRISMA. In this step, a description of the pandemic's impact on suicidal behaviour will be made, considering the descriptive variables in the studies.

Quantitative data from each study will be extracted and inserted into an Excel sheet by two independent reviewers. Statistical analyses will be carried out using the Statistical Package for the Social Sciences-SPSS, V.18.0. Standardised mean differences and $95 \%$ CI will be used to calculate the effect sizes, as we expect that most of the observational studies ${ }^{19}$ included in our review will have reported the differences in suicide behaviour.

\section{Grading the quality of evidence}

The online version of the Grading of Recommendations Assessment, Development and Evaluation (GRADE) methodology ${ }^{20}$ will be used to assess the quality of the evidence and risk of bias, categorised into four levels: high, moderate, low and very low.

\section{Patient and public involvement}

Since this is a systematic review protocol, no patients or public are involved.

\section{Ethics and dissemination}

Due to the characteristics of observational studies, an ethical assessment was not necessary. The results of this systematic review will be disseminated through publications submitted to peer review. Besides, any changes to this protocol will be documented concerning saved searches and analysis methods, which will be recorded in bibliographic databases for data collection and synthesis.

\section{DISCUSSION}

The health crisis experienced worldwide caused by the pandemic of COVID-19 has challenged the world and brought several implications, also for mental health. To cope with such a complex moment, there have been countless demands, ranging from social isolation to the overload of work at home. ${ }^{21} 22$

Thus, through rigorous screening and synthesis of the literature, it is expected that this systematic review will clarify the existence of possible factors associated with suicidal behaviour in view of the possibilities of the COVID-19 pandemic. It is important to highlight that a similar review has been developed. However, it is different from this one since it includes data from children, has broad inclusion criteria and seeks to summarise data on the incidence and the prevalence of suicidal behaviour; functional effects and effects of interventions in relation to the COVID-19 pandemic, while this proposal seeks to limit itself to know the variables related to suicidal behaviour. $^{23}$

This new context has required people, sometimes, far beyond what they can give. Thus, it is believed that psychological distress will intensify in the coming weeks and months and, consequently, suicidal behaviour presents itself as a fatal outcome related to the conflicts implied by this 'new normal'. ${ }^{24} 25$

Considering the gap in the literature that currently exists, rapid and collaborative research on the impact of the COVID-19 pandemic on suicidal behaviour is necessary and emerging since summarising epidemiological data on the impact of this pandemic on suicidal behaviour is expected to contribute to the global scientific community in the characterisation of this new virus.

It is noteworthy that the potential limitations of this systematic review may include the heterogeneity of the studies, as well as the methodological evaluation and the probably reduced number of studies, which can influence the external validity. Besides, it is expected that this review will contribute to redirecting public actions and policies aimed at tackling suicidal behaviour, especially in the peculiar context of the COVID-19 pandemic.

The realisation of this review can also stimulate other researchers to produce scientific knowledge that can effectively subsidise the clinical practice of professionals who deal with people with ideation and attempted suicide and reinforce the need for new resources aimed at preventing suicidal behaviour in the pandemic context. It is also considered essential to instigate measures to recognise demands in mental health and prevent suicide, especially in the face of a situation involving triggering aspects of psychological suffering, such as the pandemic currently experienced. In general, these strategies require scientific evidence, investments in research and recognition of the serious problem that configures suicidal behaviour. ${ }^{26}$

Twitter Priscilla Ingrid Gomes Miranda @scllm

Contributors FJGSJ and JCeSS conceptualised and designed the protocol, drafted the initial manuscript and reviewed the manuscript. FJGSJ, JCeSS, CFSM, ACMP, 
CPBV, FTG and SCP defined the concepts and search items, data extraction process as well as methodological appraisal of the studies. APCC, LRBC, LAAL, PIGM, AMSG, ARSI, CPBV, FTG and FJGSJ planned the data extraction and statistical analysis. FJGSJ, CFSM and JCSS provided critical insights. All authors have approved and contributed to the final written manuscript.

Funding The authors have not declared a specific grant for this research from any funding agency in the public, commercial or not-for-profit sectors.

Competing interests None declared.

Patient and public involvement Patients and/or the public were not involved in the design, or conduct, or reporting, or dissemination plans of this research.

Patient consent for publication Not required.

Provenance and peer review Not commissioned; externally peer reviewed.

Open access This is an open access article distributed in accordance with the Creative Commons Attribution Non Commercial (CC BY-NC 4.0) license, which permits others to distribute, remix, adapt, build upon this work non-commercially, and license their derivative works on different terms, provided the original work is properly cited, appropriate credit is given, any changes made indicated, and the use is non-commercial. See: http://creativecommons.org/licenses/by-nc/4.0/.

\section{ORCID iDs}

Fernando José Guedes da Silva Junior http://orcid.org/0000-0001-5731-632X Priscilla Ingrid Gomes Miranda http://orcid.org/0000-0001-8948-7158

\section{REFERENCES}

1 Vellas C, Delobel P, de Souto Barreto P, et al. COVID-19, virology and geroscience: a perspective. J Nutr Health Aging 2020;24:685-91.

2 World Organization Health (WHO). WHO coronavirus disease (COVID-19) Dashboard. Available: https://covid19.who.int/.

3 Esakandari $\mathrm{H}$, Nabi-Afjadi M, Fakkari-Afjadi J, et al. A comprehensive review of COVID-19 characteristics. Biol Proced Online 2020;22:2-10.

4 Werneck GL, Carvalho MS. A pandemia de COVID-19 no Brasil: crônica de uma crise sanitária anunciada. Cadernos de Saúde 2020;36:1-4.

5 Czeisler ME, Lane I, Petrosky EMD, et al. Mental health, substance use, and suicidal ideation during the COVID-19 pandemic - United States, June 24-30, 2020. Morbidity and Mortality Weekly Report 2020;69:1050-7 https://www.cdc.gov/mmwr/volumes/69/wr/pdfs/ mm6932a1-H.pdf

6 World Health Organization (WHO). Preventing suicide: a global imperative. Geneva: WHO, 2014. Available: https://www.who.int/ mental health/suicide-prevention/world report_2014/en/

7 Aquila I, Sacco MA, Ricci C, et al. The role of the COVID-19 pandemic as a risk factor for suicide: what is its impact on the public mental health state today? Psychol Trauma 2020;12:S120-2.

$8 \mathrm{Li} \mathrm{Z,} \mathrm{Ge} \mathrm{J,} \mathrm{Yang} \mathrm{M,} \mathrm{et} \mathrm{al.} \mathrm{Vicarious} \mathrm{traumatization} \mathrm{in} \mathrm{the} \mathrm{general}$ public, members, and non-members of medical teams aiding in COVID-19 control. Brain Behav Immun 2020;88:916-9.
9 Moher D, Shamseer L, Clarke M, et al. Preferred reporting items for systematic review and meta-analysis protocols (PRISMA-P) 2015 statement. Syst Rev 2015;4:1.

10 Methley AM, Campbell S, Chew-Graham C, et al. PICO, PICOS and SPIDER. a comparison study of specificity and sensitivity in three search tools for qualitative systematic reviews. BMC Health Serv Res 2014;14:579.

11 Lefebvre C, Manheimer E, Glanville J. Searching for studies. In: Higgins JPT, Greene S, eds. Cochrane Handbook for systematic reviews of interventions, version 5.0. The Cochrane Collaboration, 2008.

12 Gonçalves CA, Lopes-Júnior LC, Nampo FK, et al. Safety, efficacy and immunogenicity of therapeutic vaccines in the treatment of patients with high-grade cervical intraepithelial neoplasia associated with human papillomavirus: a systematic review protocol. BMJ Open 2019;9:e026975-5

13 Lopes-Júnior LC, Rosa MADRdeP, Lima RAGde. Psychological and psychiatric outcomes following PICU admission: a systematic review of cohort studies. Pediatr Crit Care Med 2018;19:58-67.

14 Slim K, Nini E, Forestier D, et al. Methodological index for nonrandomized studies (MINORS): development and validation of a new instrument. ANZ J Surg 2003;73:712-6.

15 Fleiss JL, Levin MPB. Statistical methods for rates and proportions. 3rd edn. John Wiley \& Sons, 2013.

16 Duval S, Tweedie R. Trim and fill: a simple funnel-plot-based method of testing and adjusting for publication bias in meta-analysis. Biometrics 2000;56:455-63.

17 Egger M, Davey Smith G, Schneider M, et al. Bias in meta-analysis detected by a simple, graphical test. BMJ 1997;315:629-34.

18 Higgins JPT, Thompson SG, Deeks JJ, et al. Measuring inconsistency in meta-analyses. BMJ 2003;327:557-60.

19 Martín-Gómez C, Moreno-Peral P, Bellón JA, et al. Effectiveness of psychological, psychoeducational and psychosocial interventions to prevent postpartum depression in adolescent and adult mothers: study protocol for a systematic review and meta-analysis of randomised controlled trials. BMJ Open 2020;10:e034424.

20 Granholm A, Alhazzani W, Møller MH. Use of the grade approach in systematic reviews and guidelines. Br J Anaesth 2019;123:554-9.

21 Bryan CJ, Bryan AO, Baker JC. Associations among state-level physical distancing measures and suicidal thoughts and behaviors among U.S. adults during the early COVID-19 pandemic. Suicide Life Threat Behav 2020;0:e12653.

22 Mejia CR, Quispe-Sancho A, Rodriguez-Alarcon JF. Factores asociados al fatalismo ante La COVID-19 en 20 ciudades del Perú en marzo 2020. Rev haban cienc méd 2020;19:1-13.

23 John A, Eyles E, McGuinness LA, et al. The impact of the COVID-19 pandemic on self-harm and suicidal behaviour: protocol for a living systematic review. F1000Res 2020;9:644

24 Brooks SK, Webster RK, Smith LE, et al. The psychological impact of quarantine and how to reduce it: rapid review of the evidence. The Lancet 2020;395:912-20.

25 Faro A, Bahiano MA, Nakano TC, et al. COVID-19 E saúde mental: a emergência do cuidado. Estudos de Psicologia 2020;37:1-14.

26 Moutier C. Suicide prevention in the COVID-19 era: transforming threat into opportunity. JAMA Psychiatry 2020. doi:10.1001/ jamapsychiatry.2020.3746. [Epub ahead of print: 16 Oct 2020]. 